\title{
Valley Vortex Assisted and Topological Protected Microparticles Manipulation with Complicated 2D Patterns in a Star-like Sonic Crystal
}

\author{
Jian Zhu *, Tianning Chen, Chen Chen and Wei Ding
}

check for updates

Citation: Zhu, J.; Chen, T.; Chen, C.; Ding, W. Valley Vortex Assisted and Topological Protected Microparticles Manipulation with Complicated 2D Patterns in a Star-like Sonic Crystal. Materials 2021, 14, 4939. https:// doi.org/10.3390/ma14174939

Academic Editor: Alina A. Manshina

Received: 9 July 2021

Accepted: 27 August 2021

Published: 30 August 2021

Publisher's Note: MDPI stays neutral with regard to jurisdictional claims in published maps and institutional affiliations.

Copyright: (c) 2021 by the authors. Licensee MDPI, Basel, Switzerland. This article is an open access article distributed under the terms and conditions of the Creative Commons Attribution (CC BY) license (https:/ / creativecommons.org/licenses/by/ $4.0 /)$.
School of Mechanical Engineering, Xi'an Jiaotong University, Xi'an 710049, China; tnchen@mail.xjtu.edu.cn (T.C.); chen2017@stu.xjtu.edu.cn (C.C.); ddj3934@stu.xjtu.edu.cn (W.D.)

* Correspondence: jianzhuxj@xjtu.edu.cn

\begin{abstract}
Arranging microparticles into desired patterns, especially in a complicated pattern with a reliable and tunable manner, is challenging but highly desirable in the fields such as biomedicine and tissue engineering. To overcome these limitations, here, by using the concept of topology in acoustics, the valley vortex is utilized to manipulate particles on a large scale with complicated 2D patterns in the star-like sonic crystals at different frequencies. A topologically protected edge state is obtained at the interface of the crystals with different valley Hall phases, which shows the ability of reliable microparticles control along the sharp corner and the capability of robust particles cluster aggregation in a defective system. The results may provide intriguing resources for future microfluidic systems in a complicated and brittle environment.
\end{abstract}

Keywords: metamaterials; topology; particle control; complicated pattern

\section{Introduction}

Precise, robust, and tunable microparticle assembly and manipulation has attracted much attention and has potential applications in numerous fields, such as biomedicine, bioanalytical chemistry, and tissue engineering [1-6]. The common optical tweezers, magnetic and electrostatic methods, etc., have successfully been used to manipulate particles, while the limitations of the weak force, high-power laser, biological sample damaging, etc., restrict their use in many applications [7-9]. An acoustic method offers an effective way to control the microparticles by using the interaction of sound with the particles and fluid inside the fluid due to its lab-free and biocompatibility [10-12]. In the field of acoustics, the most representative particle manipulation technique is the standing wave, represented by the two lab-on-chip methods, namely bulk acoustics wave (BAW) and surface acoustic wave (SAW) according to the actuation acoustic transducer. The BAW is usually generated by the piezoelectric transducer, and the SAW can be generated by the interdigitated transducer (IDTS) [6,10,11]. Many 1D (one-dimensional) alignments and 2D (two-dimensional) matrix particle patterns are designed effectively and rapidly by using one pair or two pairs of these acoustic transducers [13-15].

However, microparticle manipulation with the complicated patterns using the acousticdriven standing wave is difficult due to its inherent and unavoidable limitations, such as the design and fabrication [6]. The design and fabrication of the acoustofluidic transducer such as the ITDS in SAW devices are complicated and expensive, especially in high-frequency regions [16,17]. Further, dedicated devices, such as the high-frequency power amplifier and the signal generator, are necessary. More importantly, the higher the frequency of the acoustic wave, the higher the particle resolution. However, the wave attenuation is very fast, and the propagation distance is limited, which results in limited experimental test regions and single or small area particle manipulation.

Many other versatile acoustic wave-based methods are proposed for complicated shape particle design. For example, the monolithic acoustic holograms method is used to 
reconstruct the complex acoustic pressure fields to assemble the particles into asymmetric 2D shapes [18], the Chladni pattern formed over vibrating plates at different modes of the resonance, the hollow-shape microparticles patterning by the multimode of artificial materials [19-22], and the recent acoustic valley vortex state is also used to control and separate microparticles but at a fixed frequency and pattern [23]. The valley vortex statesbased topological edge state has attracted much attention in condensed matter physics due to the backscattering-immunity of sound transport [24-26], which has been widely used to design various acoustic wave-based devices, such as the acoustic delay line and the robust programmable waveguide [27-33]. Very recently, the edge state formed at the interface of water and the 1D arrays of Helmholtz resonant air cavities showed that it can be used to control the particle by using the oscillations [34].

To overcome the limitations, e.g., limited acoustic field patterns and small operation regions of the traditional acoustic wave-based microparticles manipulation or the vortexassisted sonic crystal at a fixed frequency, in this work, a kind of star-like sonic crystal is designed to control the particles with complicated patterns at different frequencies. Here, by using the concept of valley vortex states and protected edge states, we demonstrate that excited valley vortex states can be utilized to manipulate or assemble the particles on a large scale with complicated 2D patterns in the star-like sonic crystal at different states. In addition, a topologically protected edge state is obtained at the interface of the two types of star-like crystals, which shows the ability of reliable microparticles manipulation at the surface along the sharp corner.

\section{Structure and Design Methods}

Figure 1a shows the configuration of the 2D sonic crystal (SC). The green area represents water in our case to simulate the environment of the acoustofluidic field. The SC consisted of a hexagonal-lattice array with a three-legged scatterer in the center. The enlargement in Figure 1a on the bottom right corner shows the unit cell of the crystal. The geometry had design parameters of a lattice constant $a=43 \mathrm{~mm}$, rotate angle $\theta=0 /-5^{\circ}$, $\phi=18^{\circ}$, span $d=0.6 \mathrm{~mm}$.

Figure $1 \mathrm{~b}$ shows the calculated band structure by using the finite element method. The red dot lines represent the dispersion curve for the case of $\theta=0^{\circ}$ where the crystal had the $C_{3 v}$ symmetry, and the two Dirac points at the symmetry corner point $\mathrm{K}$ could be obtained simultaneously by controlling the scatterer and its geometrical parameters. By rotating the scatterer, for example, as indicated by the blue solid lines for the case of $\theta=5^{\circ}$, the $C_{3 v}$ symmetry of the system reduced to $C_{3}$. When the mirror symmetry was broken it resulted in the degeneracy lift as indicated by the degenerated states $p, q$, and $p^{\prime}, q^{\prime}$. Figure $1 \mathrm{c}$ shows the calculated sound field distributions at $p, q$ states for the case of rotate $\theta=-5^{\circ}$ (up parallel) and $\theta=5^{\circ}$ (bottom parallel). The black arrows represent the acoustic energy flow which shows the vortex features. The vortex had an opposite chirality, i.e., the clockwise when $\theta=5^{\circ}$ and anticlockwise when $\theta=-5^{\circ}$. The vortex chirality feature also can be observed in Figure 1d, which shows the band-edge frequencies varying with the rotation angle $\theta$ during the close and the reopening process of the bandgap. Furthermore, the different acoustic topological phases transition occurred, which can be characterized by the effective mass $m=\operatorname{sgn}\left(\omega_{q 1}-\omega_{p 1}\right)[23,28,30]$. 

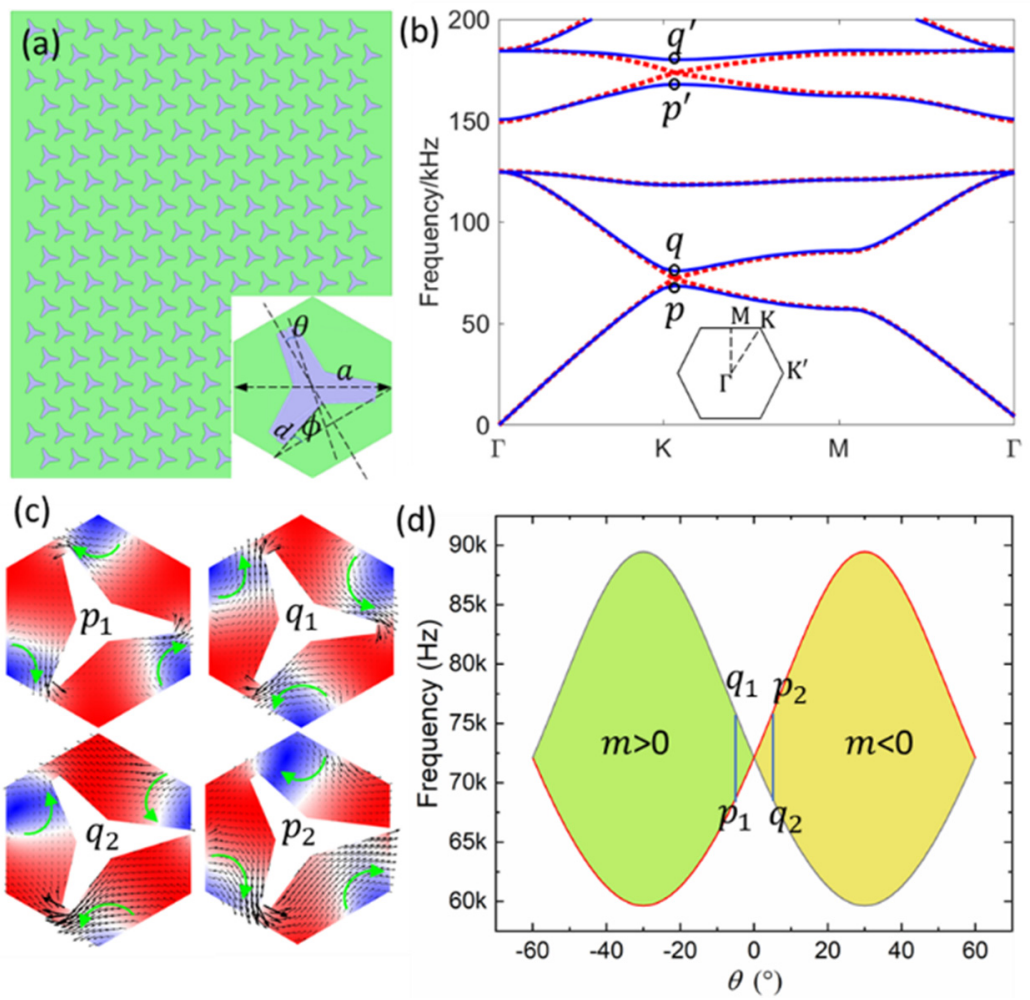

Figure 1. The configuration of the "star-like" sonic crystal and its vortex profile. (a) The sonic crystal consisted of a star-like structure embedded in the water. The enlarged unit cell of the crystal is shown in company. (b) The band structure for the case of $\theta=0^{\circ}$ (red dotted line) and $\theta=5^{\circ}$ (blue solid line) and the first Brillouin zone. (c) Calculated sound fields when $\theta=-5^{\circ}$ and $\theta=5^{\circ}$ at $p, q$ states. The black and green arrows represent the energy and vortex profiles. (d) The band-edge frequencies varied with the rotation angle $\theta$.

\section{Results}

The valley states can be excited by the external sound, and the vortex in the acoustic system can offer a new way to manipulate the transport of sound or control microparticles. In this section, the vortex features, acoustic pressure field, and the acoustic radiation force are then numerically analyzed in the 2D sonic crystal system. We demonstrate the complicated 2D patterns that can be used to manipulate particles on a large scale at different frequencies.

\subsection{D Patterns and Particles Aggregation in the Sonic Crystals at the Different States}

The valley vortex state in the crystal can be generated by the external sound excitation, and the exciting incident angle can be determined according to the conservation of the momentum parallel to the crystal interface [24]. Figure 2 shows the calculated acoustic pressure field and acoustic radiation force. In the simulation model, a plane wave excitation was applied on the left boundary. The absorbing boundary conditions were set at the top, bottom, and right boundaries to absorb the acoustic energy, as shown in Figure 2a. Moreover, the star-like scatters were assumed to be rigid wall boundaries. The largest mesh size in the simulation was smaller than one-tenth of the shortest incident wavelength. Figure 2a shows the calculated acoustic pressure field distribution when the plane wave with a frequency of $66.9 \mathrm{kHz}$ was incident from left to right. We noticed that the external sound excitation was slightly different from the theoretically predicted result in Figure $1 \mathrm{~b}$, with a maximum tolerance of $1.7 \%$ and $0.1 \%$ for $p$ and $p^{\prime}$ states, respectively. The reason why the theoretical predicted and simulated results are slightly different is due to the unavoidable uncertainty of the mismatch of the acoustic impedance [23,35] The distinct pressure nodes and antinodes were obtained, which can be used to control the trajectories 
and locations of the microparticles. The enlarged view of the field is shown in the company on the right. In Figure $2 b$, we extracted the acoustic pressure amplitudes from the green line in Figure 2a, the red dash line represents the incidence, and the blue solid line is the excited valley vortex field. The results showed that the amplitude of the excited pressure could be about 3.5 times as larger as the incidence due to the local resonance of the periodic sonic crystal structures.

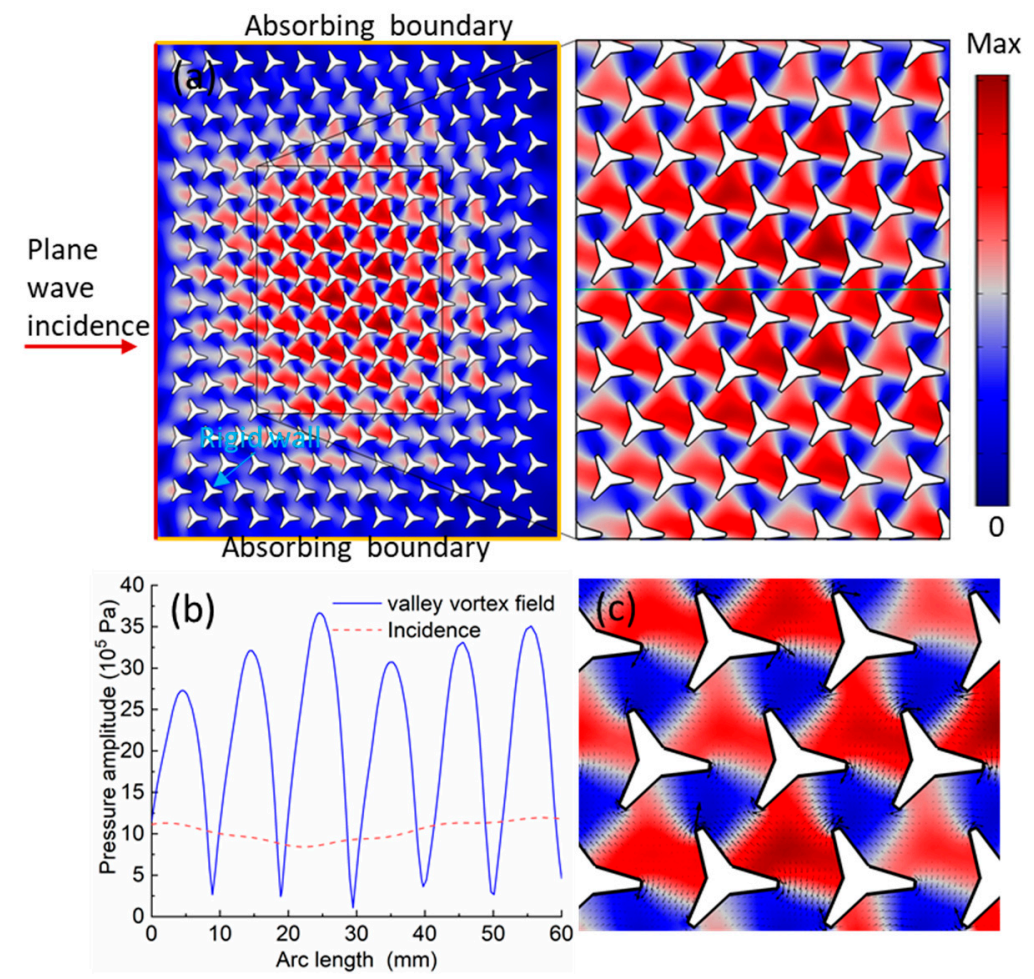

Figure 2. The acoustic field distribution and acoustic radiation force calculation. (a) The simulation model and the calculated acoustic pressure fields and its enlarged view when the plane wave was incident from left to right. (b) The extracted amplitude of the incidence (the red dash line) and the excited valley vortex field (the blue line) from the green line in (a). (c) The calculated acoustic radiation force potential and force.

After obtaining the acoustic pressure fields, we further calculated the acoustic pressureinduced acoustic radiation force according to the theory of Gor'kov $[11,36,37]$. The acoustic radiation force acting on a particle, $\boldsymbol{F}_{r}$, comes from the scattering of the ultrasound waves on the particle and is induced by radiation force potential field $U$, which can be expressed as $[37,38]$

$$
\begin{gathered}
U=V_{0}\left[f_{1} \frac{1}{4 \rho_{f} c_{f}^{2}} \operatorname{Re}\left(p \cdot p^{*}\right)-f_{2} \frac{3 \rho_{f}}{8} \operatorname{Re}\left(\boldsymbol{v} \cdot \boldsymbol{v}^{*}\right)\right] \\
\boldsymbol{F}_{\boldsymbol{r}}=-\nabla U
\end{gathered}
$$

with factors $f_{1}=1-\frac{k_{p}}{k_{f}}$ and $f_{2}=2\left(\rho_{p}-\rho_{f}\right) /\left(2 \rho_{p}+\rho_{f}\right) . V_{0}$ is the volume of the particle, $\rho_{f}$, and $k_{f}$ are the density and compressibility of the fluid, the subscripts $f$ and $p$ denote the fluid and particle, $p$ and $v$ are the calculated acoustic pressure and velocity fields.

The above problems were solved by utilizing the finite element method. The Comsol Multiphysics software was used in this case to calculate the radiation force potential field and the acoustic radiation force. The results are shown in Figure 2c, in which the 2D patterns represent the radiation force potential field, and the black arrows represent the radiation force. The direction of the arrow shows that the radiation force direction exhibited from high to low (red to the blue area) force potential field, which is an acoustic radiation characteristic. 
We can further mimic the particle distribution and locations after obtaining the acoustic radiation force in Figure 2. Here we also considered the viscous effects of the particle in the fluid. Only drag forces were considered in this work due to the calculated domain being much larger than the wavelength and the boundary layers. The drag force can be expressed as $[37,38]$

$$
F_{s}=-6 \pi \eta R\left(v_{p}-v_{f}\right)
$$

where $\eta$ and $R$ represent the viscosity of the fluid and the radius of the particle, $v_{p}$ and $v_{f}$ denote the velocity of the particle and the fluid, respectively. We assumed that the microparticles were suspended on the fluid and the surrounding background was static, and the gravity equaled to the footage.

Figure 3 shows the microparticles distribution and locations along with times when a plane wave was incident from left to right. The background fluid was water with a density $\rho_{p}=1000 \mathrm{~kg} / \mathrm{m}^{3}$, modulus $K=2.25 \mathrm{GPa}$. The particle was selected as polystyrene, which is commonly used in the field of microfluidic and polymer with physical parameters $\rho_{p}=1050 \mathrm{~kg} / \mathrm{m}^{3}$, pressure-wave speed $c_{p}=2350 \mathrm{~m} / \mathrm{s}, a=20 \mathrm{um}$. Figure 3a represents 15,000 particles (green points) that were randomly distributed in the calculated area at the starting positions when $t=0 \mathrm{~s}$. The acoustic field pattern and radiation force were obtained based on Figure 2 with the incident frequency of $66.9 \mathrm{kHz}$. The particles were gradually moved to the acoustic valley when we turned on the acoustic waves, as shown in Figure $3 b-f$, in which the distribution and locations of the particles are demonstrated at $t=1, t=2, t=3, t=4$ and $t=5 \mathrm{~s}$.

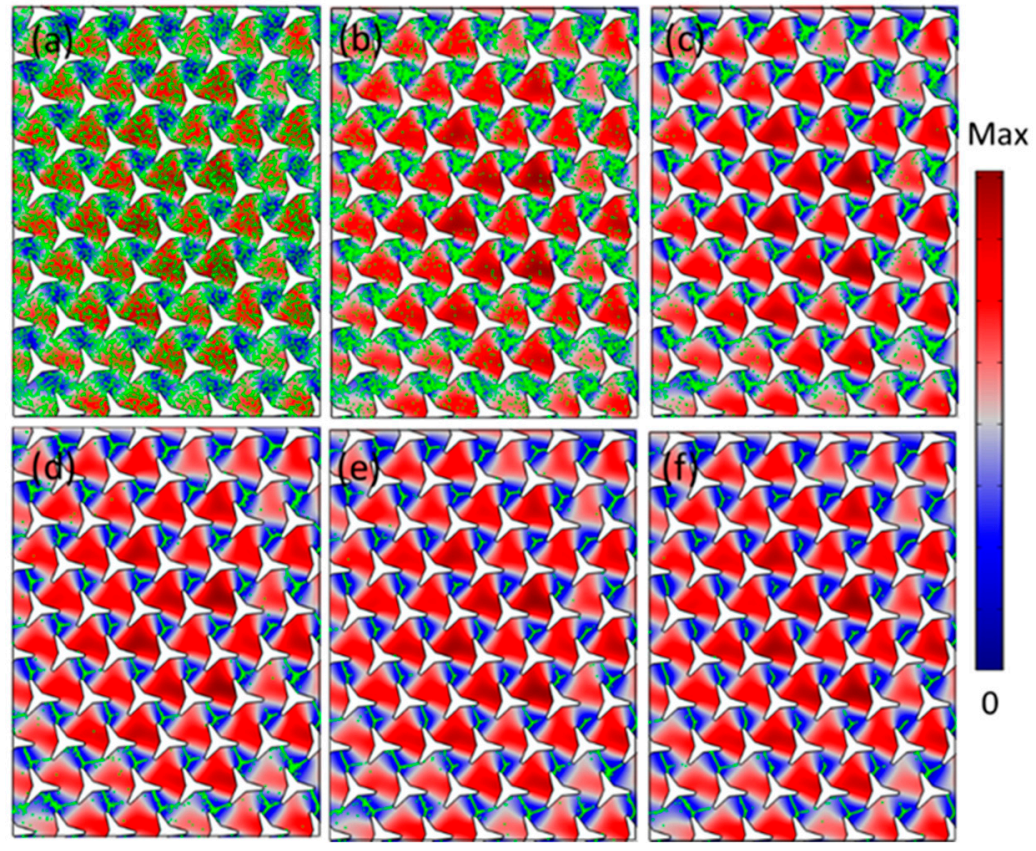

Figure 3. Numerical validation of the microparticles patterning and locations when the incident wave was excited at the frequency of $66.9 \mathrm{kHz}$. (a-f) The microparticles distribution at the stating positions when $t=0 \mathrm{~s}, t=1 \mathrm{~s}, t=2 \mathrm{~s}, t=3 \mathrm{~s}, t=4 \mathrm{~s}$, and $t=5 \mathrm{~s}$.

Further, we calculated the location tracing of the particles when the incidence at another vortex/Dirac point with the frequency at $167.6 \mathrm{kHz}$, as shown in Figure 4 . It is the particle distribution and locations when $t=0, t=1, t=2, t=3, t=5$, and $t=8 \mathrm{~s}$. Compared to the results in Figure 3, a more complicated star-like shape pattern was obtained. It cannot be easily obtained with traditional acoustofluidic methods, such as standing wave or traveling wave-based acoustic techniques. 


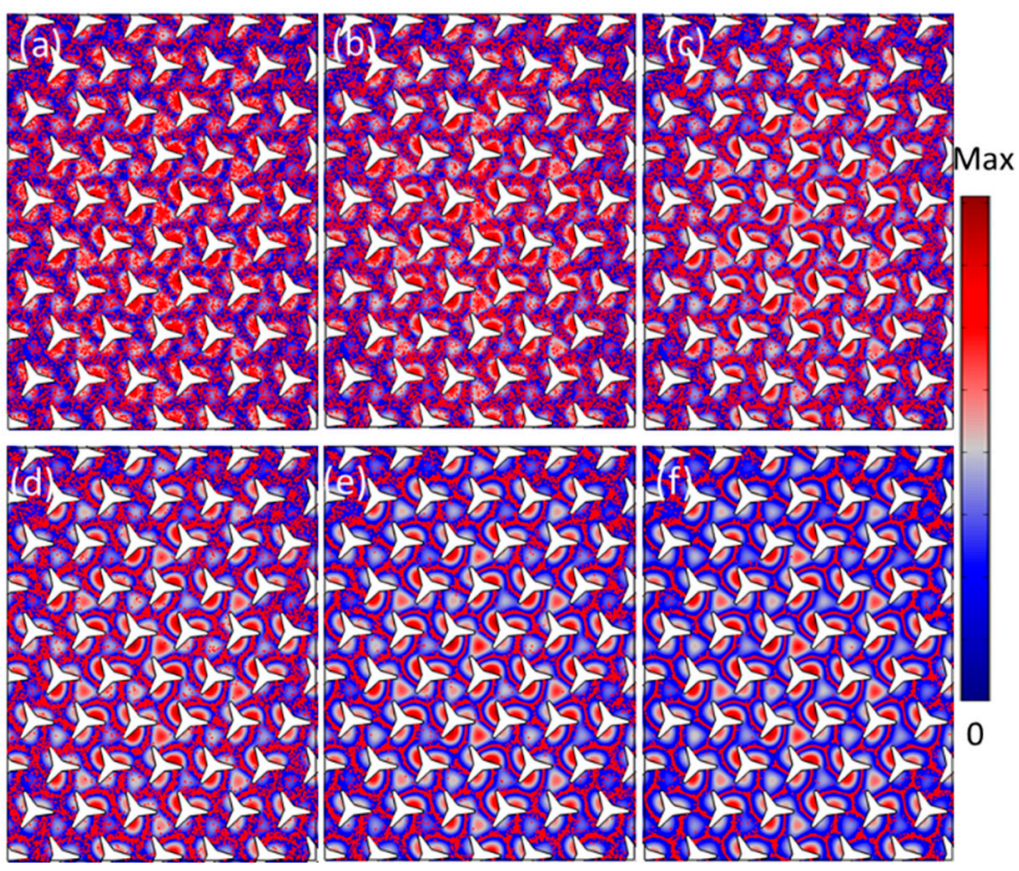

Figure 4. Numerical validation of the microparticles patterning and locations for the case of the incident wave at the frequency of $167.6 \mathrm{kHz}$. (a-f) The microparticles distribution at the stating positions when $t=0 \mathrm{~s}, t=1 \mathrm{~s} t=2 \mathrm{~s} t=3 \mathrm{~s} t=5 \mathrm{~s}$, and $t=8 \mathrm{~s}$.

\subsection{Topologically Protected Edge State and Its Microparticles Control along the Sharp Corner}

Next, we further explored the manipulation of microparticles by using the valley vortex states-based topological edge state, which owns the backscattering immunity of sound transport. The particle was selected as polydimethylsiloxane with physics parameters $\rho_{p}=965 \mathrm{~kg} / \mathrm{m}^{3}$, pressure-wave speed $c_{p}=1080 \mathrm{~m} / \mathrm{s}, a=20 \mu \mathrm{m}$.

Figure 5 shows the particle control at the edge states along the sharp corner (zigzag curve). The zigzag curve and edge state were formed at the interface of the two domains, where the two types of sonic crystals had rotation angles of $\theta=-30^{\circ}$ and $\theta=30^{\circ}$, respectively. The excitation was a point source located at $S$, as shown in Figure 5 a, in which the acoustic wave could propagate smoothly along the zigzag curve without any backscattering. The green points represent 5000 particles randomly distributed in the calculated area at the interface when $t=0 \mathrm{~s}$. Figure $5 \mathrm{~b}$ shows the particle distribution and locations when we turned on the acoustic waves when $t=1 \mathrm{~s}$. The particles were gradually moving toward the nodes of the acoustic field pattern under the acoustic radiation force and drag force. Figure $5 \mathrm{c}-\mathrm{e}$ shows the particle distribution varied with time when $t=1.5 \mathrm{~s}, t=2 \mathrm{~s}$, and $t=3 \mathrm{~s}$, respectively. An intriguing sawtooth-type particle patterning was obtained in the zigzag curve interface which was formed along the distribution of the acoustic pressure field in the channel. Figure 5e shows the robust particles cluster aggregation when $t=3 \mathrm{~s}$, even when the structures with a defect at the interface. 


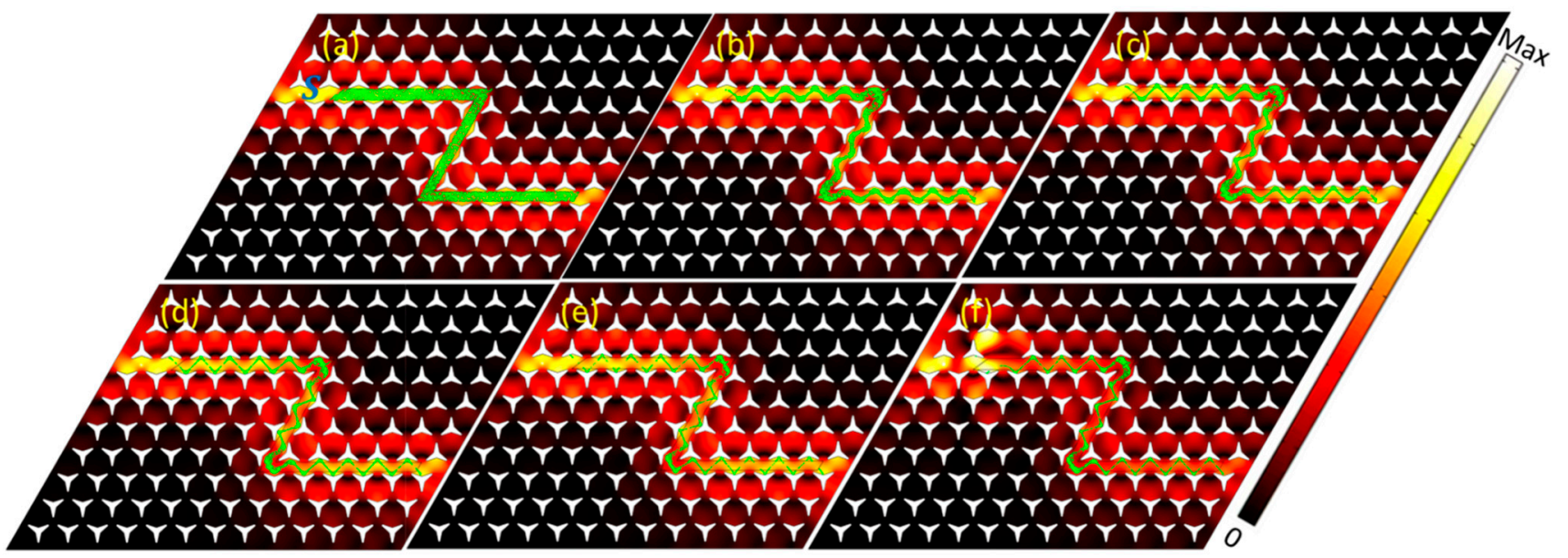

Figure 5. Microparticle distribution at the topologically protected edge states. (a) A point source was excited at $S$ with the incident frequency of $66.9 \mathrm{kHz}$, the green points are the microparticles at initial locations when $t=0 \mathrm{~s}$. (b-e) For the case of when $t=1 \mathrm{~s}, t=1.5 \mathrm{~s}, t=2 \mathrm{~s}$, and $t=3 \mathrm{~s}$. (f) For the case of $t=3 \mathrm{~s}$ with a defect at the interface of the structures.

\section{Discussion and Conclusions}

In summary, the sonic crystal (SC) is a kind of artificially periodic structure with the ability of wave or energy control in many fields, e.g., noise barrier [39,40], acoustic and vibration attenuation [41], which has attracted much attention. Here, in this work, we further constructed a type of star-like sonic crystal (SC) to show its capability of microparticle control in water application by using the concept of valley vortex states and topologically protected edge states in SC, which cannot be easily realized by using traditional acoustofluidic methods or the water-borne wave control by utilizing composite metamaterials [42,43]. The designed SC consisted of a hexagonal-lattice array with a threelegged scatterer in the center. The corresponding frequencies, bandwidth, vortex, etc., can be simply manipulated by scatterers operations. We demonstrated the excited valley vortex can be utilized to manipulate particles with complicated 2D patterns in the sonic crystals at different states. The complicated star-like shape pattern was obtained, which cannot be easily obtained with traditional acoustofluidic methods, such as standing wave or traveling wave-based acoustic devices. In addition, a topologically protected edge state was obtained at the interface of the two types of crystals, which shows the ability of reliable microparticles control along the sharp corner. Furthermore, it has the capability of robust particles cluster aggregation in a defective system. The results may promote the real applications of the acoustic topological insulators and provide an alternative way for particle manipulation in the future microfluidic system in a complicated and brittle environment.

Author Contributions: Conceptualization, J.Z.; methodology, J.Z. and T.C.; software and investigation, J.Z., C.C. and W.D.; writing — original draft preparation, J.Z.; writing—review and editing, T.C. All authors have read and agreed to the published version of the manuscript.

Funding: This work was financially funded by the National Natural Science Foundation of China (No. 12002258), the Natural Science Foundation of Shaanxi Province (No. 2020JQ-043), the China Postdoctoral Science Foundation funded project (No. 2018M643623).

Institutional Review Board Statement: Not applicable.

Informed Consent Statement: Not applicable.

Data Availability Statement: The data presented in this study are available on reasonable request from the corresponding author.

Conflicts of Interest: The authors declare no conflict of interest. 


\section{References}

1. Timonen, J.V.I.; Latikka, M.; Leibler, L.; Ras, R.H.A.; Ikkala, O. Switchable static and dynamic self-assembly of magnetic droplets on superhydrophobic surfaces. Science 2013, 341, 253-257. [CrossRef]

2. Li, H.; Torab, P.; Mach, K.E.; Surrette, C.; England, M.R.; Craft, D.W.; Thomas, N.J.; Liao, J.C.; Puleo, C.; Wong, P.K. Adaptable microfluidic system for single-cell pathogen classification and antimicrobial susceptibility testing. Proc. Natl. Acad. Sci. USA 2019, 116, 10270-10279. [CrossRef]

3. Khandurina, J.; Guttman, A. Bioanalysis in microfluidic devices. J. Chromatogr. A 2002, 943, 159-183. [CrossRef]

4. Armstrong, J.P.K.; Armstrong, J.P.; Puetzer, J.L.; Serio, A.; Guex, A.G.; Kapnisi, M.; Breant, A.; Stevens, M.M. Generating multifunctional acoustic tweezers in Petri dishes for contactless, precise manipulation of bioparticles. Adv. Mater. 2018, 30, e1802649. [CrossRef]

5. Cacace, T.; Memmolo, P.; Villone, M.M.; de Corato, M.; Mugnano, M.; Paturzo, M.; Maffettone, P.L. Assembling and rotating erythrocyte aggregates by acoustofluidic pressure enabling full phase-contrast tomography. Lab Chip 2019, 19, 3123-3132. [CrossRef] [PubMed]

6. Ozcelik, A.; Rufo, J.; Guo, F.; Gu, Y.; Li, P.; Lata, J.; Huang, T.J. Acoustic tweezers for the life sciences. Nat. Methods 2018, 15, 1021-1028. [CrossRef]

7. Chiou, P.Y.; Ohta, A.T.; Wu, M.C. Massively parallel manipulation of single cells and microparticles using optical images. Nature 2005, 436, 370-372. [CrossRef] [PubMed]

8. Hejazian, M.; Li, W.; Nguyen, N.T. Lab on a chip for continuous-flow magnetic cell separation. Lab Chip 2015, 15, 959-970. [CrossRef] [PubMed]

9. Probst, R.; Shapiro, B. Three-dimensional electrokinetic tweezing: Device design, modeling, and control algorithms. J. Micromech. Microeng. 2011, 21, 027004. [CrossRef]

10. Drinkwater, B.W. Dynamic-field devices for the ultrasonic manipulation of microparticles. Lab Chip 2016, 16, 2360-2375. [CrossRef] [PubMed]

11. Ding, X.; Li, P.; Lin, S.S.; Stratton, Z.S.; Nama, N.; Guo, F.; Slotcavage, D.; Mao, X.; Shi, J.; Costanzo, F.; et al. Surface acoustic wave microfluidics. Lab Chip 2013, 13, 3626-3649. [CrossRef] [PubMed]

12. Devendran, C.; Albrecht, T.; Brenker, J.; Alan, T.; Neild, A. The importance of travelling wave components in standing surface acoustic wave (SSAW) systems. Lab Chip 2016, 16, 3756-3766. [CrossRef] [PubMed]

13. Arrabito, G.; Galati, C.; Castellano, S.; Pignataro, B. Luminometric sub-nanoliter droplet-to-droplet array (LUMDA) and its application to drug screening by phase I metabolism enzymes. Lab Chip 2013, 13, 68-72. [CrossRef]

14. Tian, L.; Martin, N.; Bassindale, P.G.; Patil, A.J.; Li, M.; Barnes, A.; Mann, S. Spontaneous assembly of chemically encoded two-dimensional coacervate droplet arrays by acoustic wave patterning. Nat. Commun. 2016, 7, 1-10.

15. Collins, D.J.; O’Rorke, R.; Neild, A.; Han, J.; Ai, Y. Acoustic fields and microfluidic patterning around embedded micro-structures subject to surface acoustic waves. Soft Matter 2019, 15, 86918705. [CrossRef]

16. Chen, W.; Holm, S. Modified Szabo's wave equation models for lossy media obeying frequency power law. J. Acoust. Soc. Am. 2003, 114, 2570-2574. [CrossRef] [PubMed]

17. Zhou, Y.; Ma, Z.; Ai, Y. Submicron particle concentration and patterning with ultralow frequency acoustic vibration. Anal. Chem. 2020, 92, 12795-12800. [CrossRef]

18. Melde, K.; Mark, A.G.; Qiu, T.; Fischer, P. Holograms for acoustics. Nature 2016, 537, 518-522. [CrossRef] [PubMed]

19. Lei, J.; Cheng, F.; Liu, G.; Li, K.; Guo, Z. Dexterous formation of unconventional Chladni patterns using standing bulk acoustic waves. Appl. Phys. Lett. 2020, 117, 184101. [CrossRef]

20. Dai, H.; Xia, B.; Yu, D. Acoustic patterning and manipulating microparticles using phononic crystal. J. Phys. D Appl. Phys. 2019, 52, 425302. [CrossRef]

21. Fard, S.M.; Peters, H.; Kessissoglou, N.; Marburg, S. Three-dimensional analysis of a noise barrier using a quasi-periodic boundary element method. J. Acoust. Soc. Am. 2015, 137, 3107-3114. [CrossRef] [PubMed]

22. Tang, Q.; Liu, P.; Guo, X.; Zhou, S.; Dong, Y. 2D acoustofluidic patterns in an ultrasonic chamber modulated by phononic crystal structures. Microfluid. Nanofluid. 2020, 24,1-22. [CrossRef]

23. Dai, H.; Chen, T.; Jiao, J.; Xia, B.; Yu, D. Topological valley vortex manipulation of microparticles in phononic crystals. J. Appl. Phys. 2019, 126, 145101. [CrossRef]

24. Lu, J.; Qiu, C.; Ke, M.; Liu, Z. Valley vortex states in sonic crystals. Phys. Rev. Lett. 2016, 116, 093901. [CrossRef] [PubMed]

25. Ma, G.; Xiao, M.; Chan, C.T. Topological phases in acoustic and mechanical systems. Nat. Rev. Phys. 2019, 1, 281-294. [CrossRef]

26. Lu, J.; Qiu, C.; Deng, W.; Huang, X.; Li, F.; Zhang, F.; Chen, S.; Liu, Z. Valley topological phases in bilayer sonic crystals. Phys. Rev. Lett. 2018, 120, 116802. [PubMed]

27. Zhang, Z.; Tian, Y.; Cheng, Y.; Wei, Q.; Liu, X.; Christensen, J. Topological acoustic delay line. Phys. Rev. Appl. 2018,9 , 034032. [CrossRef]

28. Geng, Z.G.; Peng, Y.G.; Shen, Y.X.; Zhao, D.G.; Zhu, X.F. Acoustic delay-line filters based on largely distorted topological insulators. Appl. Phys. Lett. 2018, 113, 033503. [CrossRef]

29. Lu, J.; Qiu, C.; Ye, L.; Fan, X.; Ke, M.; Zhang, F.; Liu, Z. Observation of topological valley transport of sound in sonic crystals. Nat. Phys. 2017, 13, 369-374. 
30. Chen, C.; Chen, T.; Wang, Y.; Wu, J.; Zhu, J. Observation of topological locally resonate and Bragg edge modes in a two-dimensional slit-typed sonic crystal. Appl. Phys. Express 2019, 12, 097001. [CrossRef]

31. Geng, Z.G.; Peng, Y.G.; Li, P.Q.; Shen, Y.X.; Zhao, D.G.; Zhu, X.F. Mirror-symmetry induced topological valley transport along programmable boundaries in a hexagonal sonic crystal. J. Phys. Condens. Matter 2019, 31, 245403. [CrossRef]

32. Xia, J.P.; Jia, D.; Sun, H.X.; Yuan, S.Q.; Ge, Y.; Si, Q.R.; Liu, X.J. Programmable coding acoustic topological insulator. Adv. Mater. 2018, 30, 1805002. [CrossRef]

33. Chen, C.; Chen, T.; Song, A.; Song, X.; Zhu, J. Switchable asymmetric acoustic transmission based on topological insulator and metasurfaces. J. Phys. D Appl. Phys. 2020, 53, 44LT01. [CrossRef]

34. Dai, H.; Liu, L.; Xia, B.; Yu, D. Experimental realization of topological on-chip acoustic tweezers. Phys. Rev. Appl. 2021, 15, 064032. [CrossRef]

35. Sharma, G.S.; Faverjon, B.; Dureisseix, D.; Skvortsov, A.; MacGillivray, I.; Audoly, C.; Kessissoglou, N. Acoustic performance of a periodically voided viscoelastic medium with uncertainty in design parameters. J. Vib. Acous. 2020, 142, 061002. [CrossRef]

36. Gor'kov, L.P. On the forces acting on a small particle in an acoustical field in an ideal fluid. Sov. Phys. Dokl. 1962, 6, 773-775.

37. Bruus, H. Acoustofluidics 7: The acoustic radiation force on small particles. Lab Chip 2012, 12, 1014-1021. [CrossRef] [PubMed]

38. Riaud, A.; Wang, W.; Thai, A.L.; Taly, V. Mechanical characterization of cells and microspheres sorted by acoustophoresis with in-line resistive pulse sensing. Phys. Rev. Appl. 2020, 13, 034058. [CrossRef]

39. Martinez-Sala, R.; Sancho, J.; Sanchez, J.V.; Gomez, V.; Llinares, J.; Meseguer, F. Sound attenuation by sculpture. Nature 1995, 378, 241. [CrossRef]

40. Sanchez-Dehesa, J.; Garcia-Chocano, V.M.; Torrent, D.; Cervera, F.; Cabrera, S. Noise control by sonic crystal barriers made of recycled materials. J. Acoust. Soc. Am. 2011, 129, 1173-1183. [CrossRef] [PubMed]

41. Elmadih, W.; Chronopoulos, D.; Zhu, J. Metamaterials for simultaneous acoustic and elastic bandgaps. Sci. Rep. 2021, 11, 1-10. [CrossRef] [PubMed]

42. Sharma, G.S.; Skvortsov, A.; MacGillivray, I.; Kessissoglou, N. On superscattering of sound waves by a lattice of disk-shaped cavities in a soft material. Appl. Phys. Lett. 2020, 116, 041602. [CrossRef]

43. Sharma, G.S.; Skvortsov, A.; MacGillivray, I.; Kessissoglou, N. Sound scattering by a bubble metasurface. Phys. Rev. B 2020, 102, 214308. [CrossRef] 